\title{
2
}

\section{Illinois Transient Model: Simulating the Flow Dynamics in Combined Storm Sewer Systems}

\author{
Arturo S. Leon, Nils Oberg, Arthur R. Schmidt and Marcelo H. Garcia
}

This chapter describes the capabilities and features of the recently developed Illinois transient model (ITM) for simulating the flow dynamics (transient and non-transient conditions) in combined storm sewer systems, ranging from dry bed flows, to gravity flows, to partly gravity-partly surcharged flows (mixed flows), to fully pressurized flows (water hammer flows). ITM, which was originally developed at the University of Illinois at UrbanaChampaign, is a finite volume (FV) model that can handle complex boundary conditions such as drop shafts, reservoirs, closing and opening of gates as a function of time, and junctions with any number of connecting pipes and any types of horizontal and vertical alignment. ITM is open source code that is in constant development and its releases are made available on a regular basis. In the current version of ITM (v. 1.3, September 2010), the free surface region is modeled using the one dimensional (1-D) Saint-Venant equations. The pressurized region is modeled using the 1-D compressible water hammer equations. Open channel-pressurized flow (mixed flow) interfaces are modeled by enforcing mass, momentum and energy relations across the interfaces together with Riemann solvers at the sides of mixed flow interfaces. This version of ITM is referred to as the two equation model. The current version of ITM is superior to other models of its kind because it is robust, can simulate mixed flows (simultaneous occurrence of free surface and pressurized flows) when using actual pressure wave celerities $(\sim 1000 \mathrm{~m} / \mathrm{s})$, and because no Preissmann slot assumption is made to simulate pressurized flows (water hammer flows). ITM has been applied to

Leon, A., N. Oberg, A.R. Schmidt and M.H. Garcia. 2011. "Illinois Transient Model: Simulating the Flow Dynamics in Combined Storm Sewer Systems." Journal of Water Management Modeling R241-02. doi: 10.14796/JWMM.R241-02.

(C) CHI 2011 www.chijournal.org ISSN: 2292-6062 (Formerly in Cognitive Modeling of Urban Water Systems. ISBN: 978-0-9808853-4-7) 
several existing combined sewer systems in the U.S. and around the world, to improve the understanding of the flow dynamics of these systems.

\subsection{Introduction}

\subsubsection{ITM History and its Modeling Capabilities}

ITM was first developed in 2004 using a modified Preissmann slot approach (one governing equation) for simulating mixed flows. The 2004 version of ITM (like other Preissmann slot based models) is unable to simulate negative pressures and may lead to large mass errors when a relatively wide slot is used (typically, for avoiding numerical instabilities). Because negative pressures are very common in pressurized transient flows, it was decided to change the approach for handling mixed flows. The second version of ITM was completed in 2006. In that version the free surface region is modeled using the 1-D Saint-Venant equations; the pressurized region is modeled using the 1-D compressible water hammer equations; and open channelpressurized flow interfaces are modeled by enforcing mass, momentum and energy relations across the interfaces together with Riemann solvers at the sites of the mixed flow interfaces. This version of ITM is called the two equation model. The current version of ITM (v. 1.3, September 2010) is an improved version of the 2006 ITM model.

The current version of ITM has features that make this model superior to other models of its kind for analyzing transient flows in complex closedconduit systems. The first feature is that ITM can simulate all possible flow regimes in complex closed-conduit systems. In particular, ITM can accurately describe positive and negative open channel-pressurized flow interfaces, interface reversals, and it can simulate sub-atmospheric pressures in the pressurized flow region. Song et al. (1983) defined an open channelpressurized (mixed) flow interface as positive if it is moving towards the open channel flow and negative or retreating if it is moving towards the region of pressurized flow. The change in direction of the interface from positive to negative is called interface reversal. The second feature is that ITM can simulate transient mixed flows when large pressure wave celerities $(\sim 1000 \mathrm{~m} / \mathrm{s})$ are used. The latter is very important when pressurized transient flows are of interest. If capturing pressure transients (e.g., due to flow compressibility) are of no interest, a small pressure wave celerity may be used to speed up the computations. Furthermore, for open channel flows ITM can simulate subcritical and supercritical flows.

The graphical user interface of ITM was modified from the graphical user interface of the storm water management model (SWMM) originally 
developed by the U.S. EPA. SWMM has a powerful graphical user interface but is a simplified hydraulic model that has limitations for analyzing hydraulic transients. To take advantage of the tools of the SWMM graphical user interface, the graphical user interface of ITM was adapted from the graphical user interface of SWMM.

\subsubsection{Common Applications and Limitations of ITM}

ITM has been used in several studies. These include the assessment of the impact of gate closures in the generation of transients in combined sewer systems and the study of transient phenomena and conveyance capacity in combined sewer systems associated with heavy rainfall events.

Overall, ITM can be used for simulating all possible flow regimes in complex closed-conduit systems ranging from dry bed flows to gravity flows, to partly gravity-partly surcharged flows (mixed flows), to fully pressurized flows. The boundary conditions currently supported include drop shafts, reservoirs, rating curves, constant flow or pressure, closing and opening of gates in pressurized flow conditions as a function of time, and junctions with any number of connecting pipes and any type of horizontal and vertical alignment.

ITM has the intrinsic limitations of a 1-D model. However, it has been shown in various publications that ITM can predict with good accuracy pressure and flow discharge fluctuations for free surface, pressurized and mixed flows conditions. The current version of the ITM model is also limited to conduits of circular cross section.

\subsection{Governing Equations, Numerical Techniques and Validation of ITM}

\subsubsection{Governing Equations}

The 1-D open channel and compressible water hammer flow continuity and momentum equations for prismatic conduits are written in their vector conservative form (Guinot, 2003; Leon, 2006; Leon et al., 2006; 2008):

$$
\frac{\partial U}{\partial t}+\frac{\partial F}{\partial x}=S
$$

where the vector variable $\mathbf{U}$, the flux vector $\mathbf{F}$ and the source term vector $\mathbf{S}$ for open channel flows may be written (Leon 2006, Leon et al. 2006): 


$$
\mathrm{U}=\left[\begin{array}{l}
\rho A \\
\rho Q
\end{array}\right], \mathrm{F}=\left[\begin{array}{c}
\rho Q \\
\rho \frac{Q^{2}}{A}+A \bar{p}
\end{array}\right] \text {, and } \mathrm{S}=\left[\begin{array}{c}
0 \\
\left(S_{0}-S_{e}\right) \rho g A
\end{array}\right]
$$

where the variables for free surface flows are:

$A=$ cross-sectional area of the flow,

$Q=$ flow discharge,

$\bar{p}=$ average pressure of the water column over the cross sectional area,

$\rho=$ liquid density (assumed constant for free surface flows but not for pressurized flows),

$g=$ gravitational acceleration,

$S_{o}=$ bottom slope of the conduit, and

$S_{e}=$ slope of the energy line.

For compressible water hammer flows, the vectors may be written (Guinot, 2003; Leon, 2006; Leon et al., 2008):

$$
U=\left[\begin{array}{c}
\rho_{f} A \\
\rho_{f} Q
\end{array}\right], F=\left[\begin{array}{c}
\rho_{f} Q \\
\rho_{f} \frac{Q^{2}}{A_{f}}+A_{f} p
\end{array}\right] \text { and } S=\left[\begin{array}{c}
0 \\
\left(S_{0}-S_{e}\right) \rho_{f} g A_{f}
\end{array}\right]
$$

where:

$$
\begin{aligned}
A_{f} & =\text { full cross-sectional area of the conduit, } \\
p & =\text { pressure acting on the center of gravity of } A_{f}, \text { and } \\
\rho_{f} & =\text { liquid density for compressible water hammer } \\
& \text { flows. }
\end{aligned}
$$

Equation 2.1 is for compressible water hammer flows that do not form a closed system in that the flow is described using the three variables $\rho_{f}, p$ and $Q$. However, it is possible to eliminate the pressure variable by using the general definition of the pressure wave celerity $a_{g}$, which relates $p$ and $\rho_{f}$ (Guinot 2003):

$$
a_{g}=\left[\frac{d\left(A_{f} p\right)}{d\left(A_{f} p_{f}\right)}\right]^{1 / 2}
$$

The wave celerity in single phase (pure liquid) pressurized flows, $a$, is assumed to be constant and can be estimated using the following relation that is derived from classical structural mechanics (Wylie and Streeter, 1983):

$$
a=\left[\frac{k_{f} / \rho_{r e f}}{1+\frac{k_{f}}{E} \frac{d}{e}}\right]^{1 / 2}
$$


where:

$$
\begin{aligned}
\rho_{r e f} & =\text { reference density } \\
d & =\text { pipe diameter, } \\
E & =\text { Young's modulus of elasticity of the pipe material, } \\
e & =\text { wall thickness, and } \\
k_{f} & =\text { compressibility of the fluid in the pipe. }
\end{aligned}
$$

Assuming an infinitely rigid pipe (i.e. $A_{f}$ is constant) and substituting $a_{g}$ with $a$ in Equation 2.4, the integration of the differentials $d \rho_{f}$ and $d p$ gives the following equation relating $p$ and $\rho_{f}$ (Leon et al., 2007; 2008):

$$
p=p_{r e f}+a^{2}\left(\rho_{f}-\rho_{r e f}\right)
$$

where:

$$
p_{\text {ref }}=\text { reference pressure. }
$$

In free surface flows, the gravity wavespeed $c$ is given by $c=\sqrt{g A / T}$, where $T$ is the topwidth of the flow. According to this relation, the gravity wavespeed is unbounded as the water depth approaches the crown of the conduit. When the water depth approaches the crown of the conduit, the pressure wave and not the gravity wave should become the primary mode of propagation of a disturbance. In ITM, the phase change from free surface to pressurized flow (not from pressurized to free surface flow) is assumed to

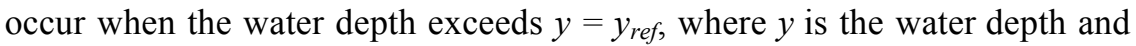
$y_{\text {ref }}$ is a reference depth. At this threshold condition $y=y_{\text {ref, }}$ all the flow parameters (fluid density, hydraulic area and average pressure) in both the open channel and the pressurized flow regime have to be the same. The results are not sensitive to $y_{\text {ref }}$. However, when using a small value of $y_{\text {ref }}$ large mass conservation errors may occur. The reference area $A_{r e f}$ is the hydraulic area below $y_{\text {ref }}$, and $A_{f}$ in the previous equations is replaced with $A_{\text {ref }}$. When using ITM to simulate pure pressurized flows, $A_{\text {ref }}$ can be set equal to $A_{f}$. The assumed reference density $\rho_{\text {ref }}$ is set to $1000 \mathrm{~kg} / \mathrm{m}^{3}$ which corresponds to clean water at $4{ }^{\circ} \mathrm{C}$. For the phase change from pressurized to free surface flow (depressurization) the criteria given in Yuan (1984) are used.

\subsubsection{Numerical Solution of Governing Equations}

The numerical scheme used in ITM is an explicit FV Godunov type method. FV methods have the ability to capture discontinuities (e.g. shocks) in the solution automatically, without explicitly tracking them (Toro, 2001). The FV method is based on writing the governing equations in integral form over an elementary control volume or cell, hence the term finite volume method. The computational grid or cell involves discretization of the spatial domain $x$ into cells of length $\Delta x$ and the temporal domain $t$ into intervals of duration 
$\Delta t$. The $i$ th cell is centered at node $i$ and extends from $i-1 / 2$ to $i+1 / 2$. The flow variables $A$ and $Q$ are defined at the cell center $i$ and represent the average value within each cell. Fluxes, on the other hand are evaluated at the interfaces between cells $(i-1 / 2$ and $i+1 / 2)$. For the $i$ th cell, the updating FV formula for the left side of Equation 2.1 is given by Toro, 2001:

$$
U_{i}^{n+1}=U_{i}^{n}-\frac{\Delta t}{\Delta x_{i}}\left(F_{i+1 / 2}^{n}-F_{i-1 / 2}^{n}\right)
$$

where: $n$ and $n+1$ reflect the $t$ and $t+\Delta t$ time levels, respectively.

In Equation 2.7, the determination of $\mathbf{U}$ at the new time step $n+1$ requires computation of the numerical flux $\mathbf{F}$ at the cell interfaces at the old time $n$. To introduce the source terms (right side of Equation 2.1) into the solution, a first order time splitting method is used which takes into account an algorithm for ensuring that stationary flows do not produce physically impossible flows. In the Godunov approach, the flux $F_{i+1 / 2}^{n}$ is obtained by solving the Riemann problem with constant states $U_{i}^{n}$ and $U_{i}^{n+1}$. This way of computing the flux leads to a first order accuracy of the numerical solution.

To achieve a second-order accuracy in space and time in ITM, the MUSCL-Hancock method (Toro 2001) is used. Second- or higher-order schemes are prone to spurious oscillations in the vicinity of discontinuities. To preserve the second-order accuracy of the solution away from discontinuities, while ensuring that the solution is oscillation-free near shock waves and other sharp flow features, a total variation-diminishing (TVD) method was used in ITM. The TVD property of the MUSCL-Hancock method is ensured by applying the MINMOD pre-processing slope limiter (see Toro, 2001). For a comprehensive description of the numerical method used in ITM for free surface, pressurized and mixed flows, see Leon (2006) and Leon et al. (2006; 2008; 2010).

With regard to the boundary conditions (BCs), the BCs currently supported by ITM include drop shafts, reservoirs, constant flow or pressure, rating curves, closing and opening of gates in pressurized flow conditions as a function of time, and junctions with any number of connecting pipes and any type of horizontal or vertical alignment. Leon et al. (2009b) presents a general boundary condition for transient flows in a drop shaft connected to an arbitrary number of pipes. This BC is general in the sense that it handles all possible flow regimes and their combinations at a junction.

\subsubsection{Validation of ITM}

ITM has been validated with experimental measurements for single pipe setups. For complex test cases (e.g. complex boundaries), ITM was validated 
with computational fluid dynamics (CFD) modeling results because of the lack of experimental data under these conditions. See Leon (2006), Leon et al. (2006; 2008; 2009a; 2010) and Leon et al. (2009b) for a description of all test cases used for validating ITM.

\subsection{Example of Application of ITM}

In this section, ITM is applied to a hypothetical closed-conduit system to provide an insight in setting input data, and running and visualizing results when using this model. For an in-depth discussion of these tasks see the user's manual of ITM (Leon and Oberg 2010). The hypothetical closedconduit system, a plan of which is shown in Figure 2.1, consists of reservoir A, fifteen tunnel reaches and eleven drop shafts with inflow hydrographs. The stage-storage curve of reservoir A is shown in Figure 2.2. Eleven inflow hydrographs were specified for this hypothetical system; one of these is shown in Figure 2.3. In the current version of ITM all drop shafts are assumed to have an infinite height. Future experimental and numerical research on overflows will allow a better simulation of the overflows and its return to the tunnel system. The input files used for this hypothetical system as well as the executable and manuals of ITM are available at the link $\mathrm{http}: / /$ web.engr.oregonstate.edu/ leon/ITM.htm

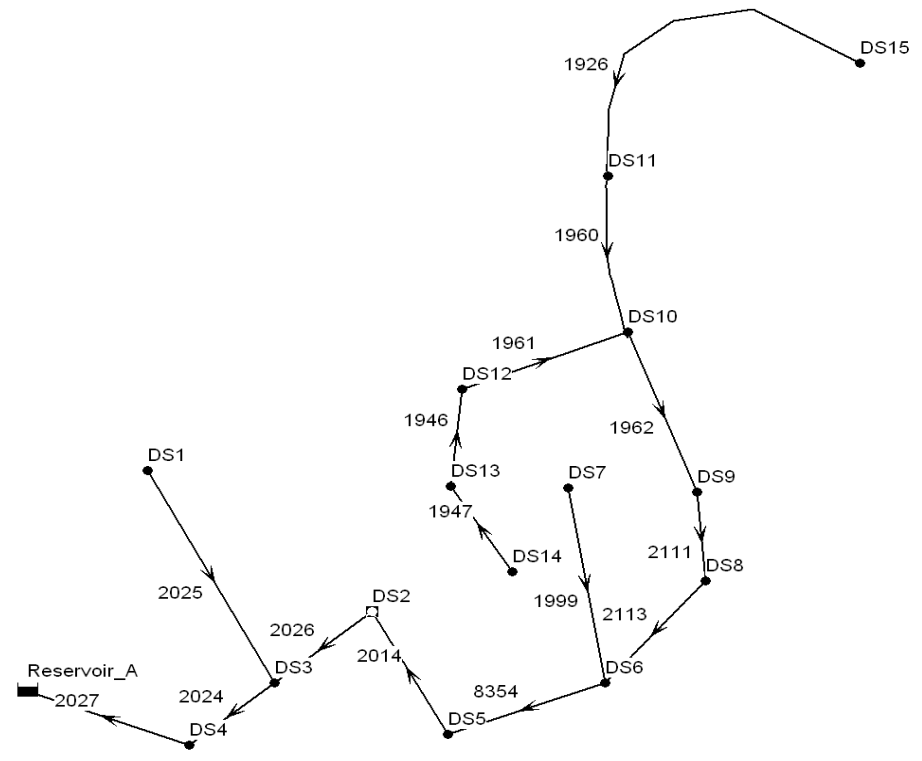

Figure 2.1 Layout of the hypothetical system showing the links (conduits) and nodes. 


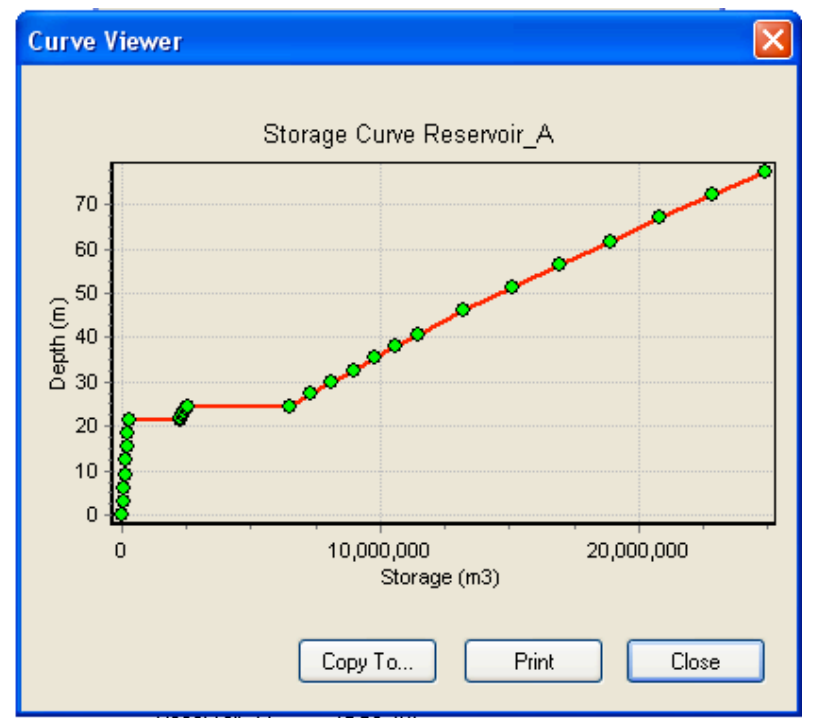

Figure 2.2 Stage-storage curve of reservoir A.

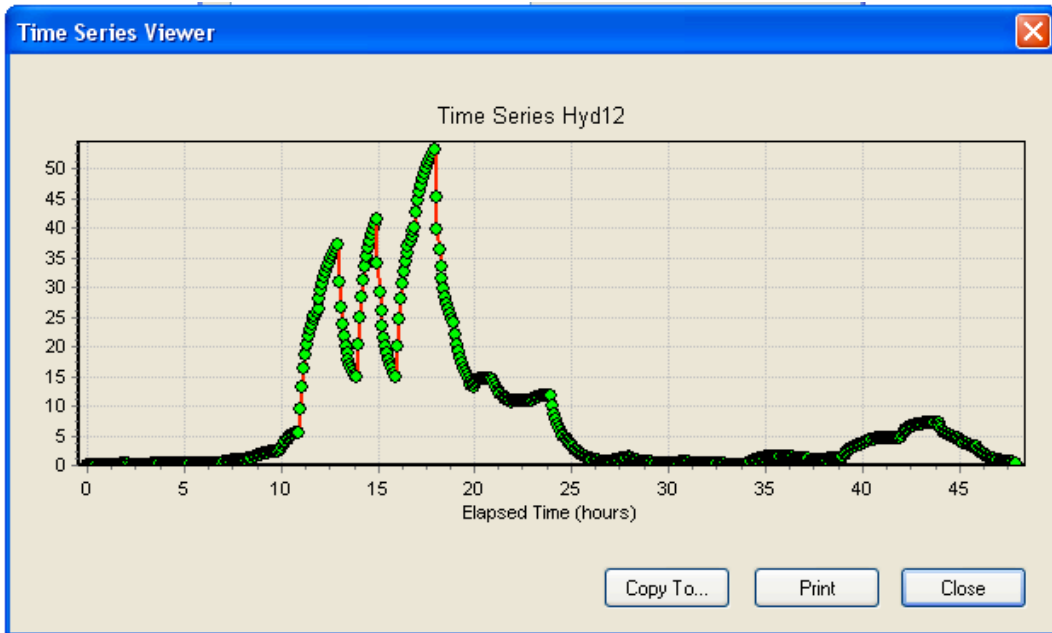

Figure 2.3 One of the inflow hydrographs used for this example.

As mentioned earlier, ITM is intended for transient and non-transient flow conditions. To show that ITM can handle large pressure wave celerities in mixed flow conditions, the pressure wave celerity used in the present simulations was set to $1000 \mathrm{~m} / \mathrm{s}$. An initial dry bed state (zero flow depth and discharge) was used as an initial condition. 
Due to data storage limitations when simulating transient flows, usually different output times are used. A coarse output time is first used to locate the periods at which transients occur in the system. Once the periods of transients are identified, a fine output time is used but the reporting period is limited to the period of the transients. A fine output time is used to accurately capture the peaks of the pressure oscillations. When the data storage becomes unmanageable, especially when long periods are simulated, the HOTSTART option of ITM may be used. The HOTSTART option allows saving the data of the last time step of a simulation and use this data as initial conditions for a new simulation. The ITM simulation options, which are located within the simulation options component, contain the parameters used for the pressure wave celerity, and those that define the accuracy of the simulation. Figure 2.4 shows the ITM options used in this example.

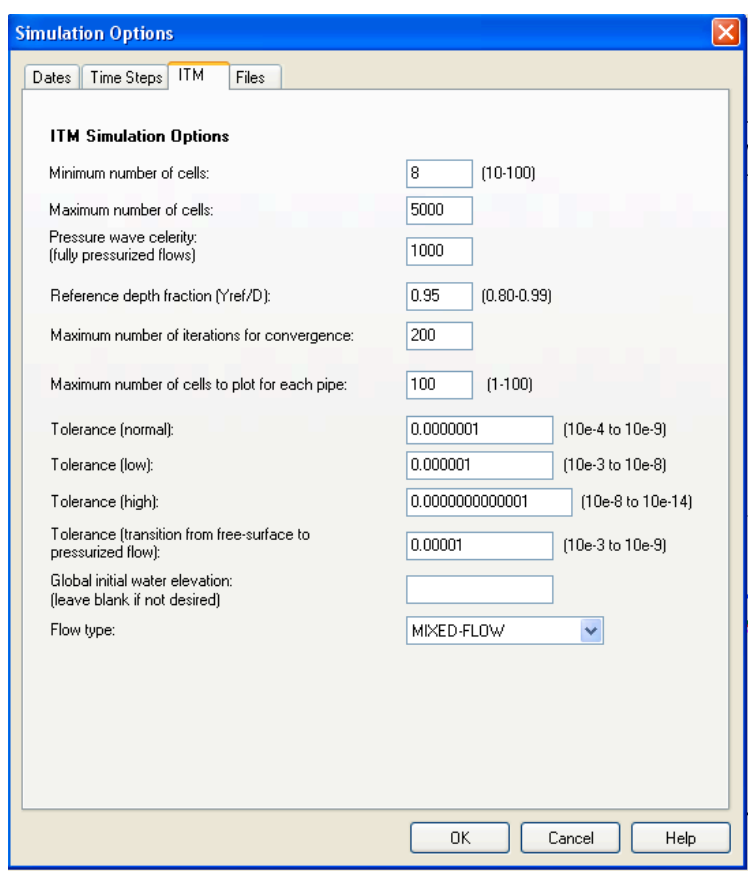

Figure 2.4 ITM parameters in the simulation options.

As mentioned earlier, the user interface of ITM was modified from the user interface of the SWMM model and it has inherited many of the SWMM features. ITM (in a similar way to the SWMM model) can plot pressure heads (piezometric elevation) and piezometric depths (measured from pipe invert) at any node, and piezometric depth, velocity and flow discharge traces at any conduit or link. As an example, the simulation results for the 
piezometric depth traces at various nodes are presented in Figure 2.5. For the links, the values reported in ITM are those at the center of the conduit (not the average of the conduit). The simulation results for the piezometric depth, velocity and flow discharge traces at the center of various conduits (links) for a coarse output time are presented in Figures 2.6, 2.7 and 2.8, respectively. A negative flow discharge or velocity indicates that the flow direction is in the opposite direction to the flow in normal conditions (in normal conditions, flow is from high to low elevation).

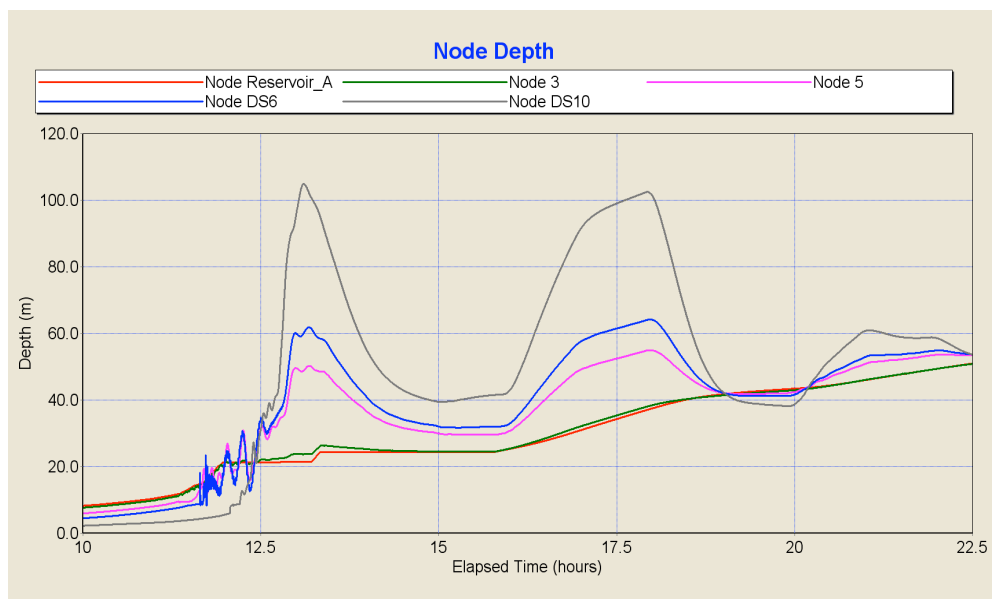

Figure 2.5 Piezometric depth traces at various nodes for the coarse output time (Initial dry bed state, $a=1000$ ).

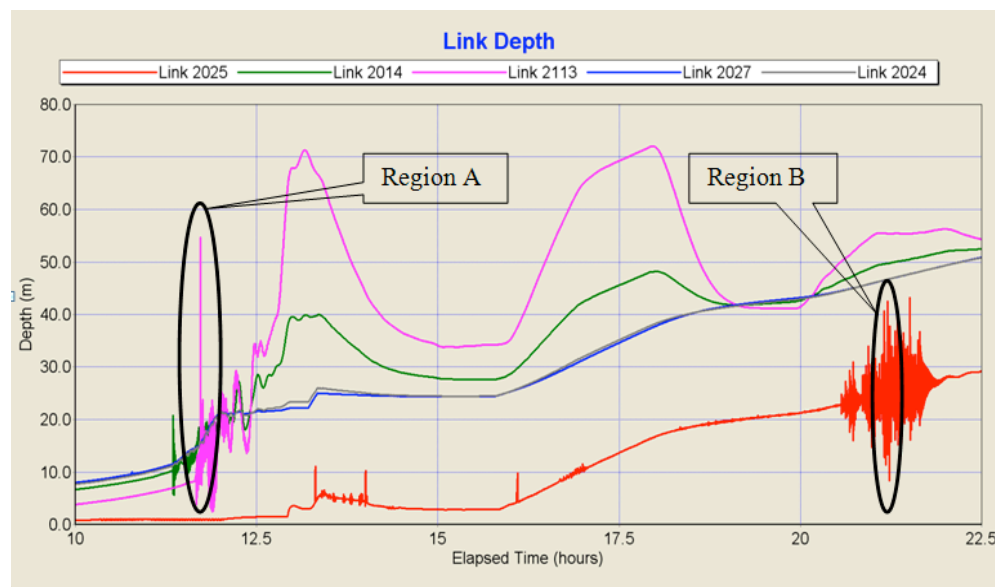

Figure 2.6 Piezometric depth traces at the center of various tunnel reaches for the coarse output time (Initial dry bed state, $a=1000$ ). 
33 Illinois Transient Model: Simulating the Flow Dynamics in CSS Systems

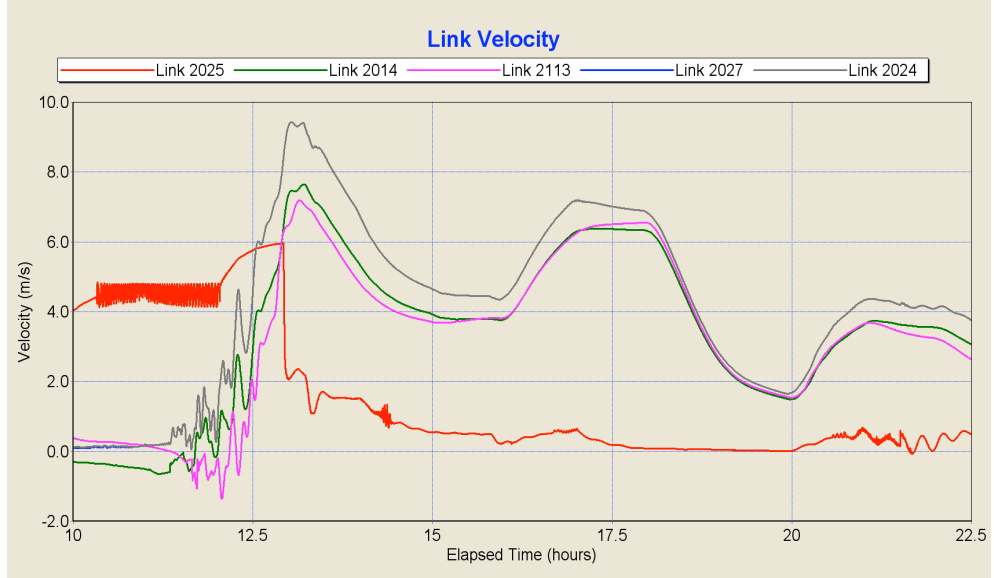

Figure 2.7 Flow velocity traces at the center of various tunnel reaches for the coarse output time (Initial dry bed state, $a=1000$ ).

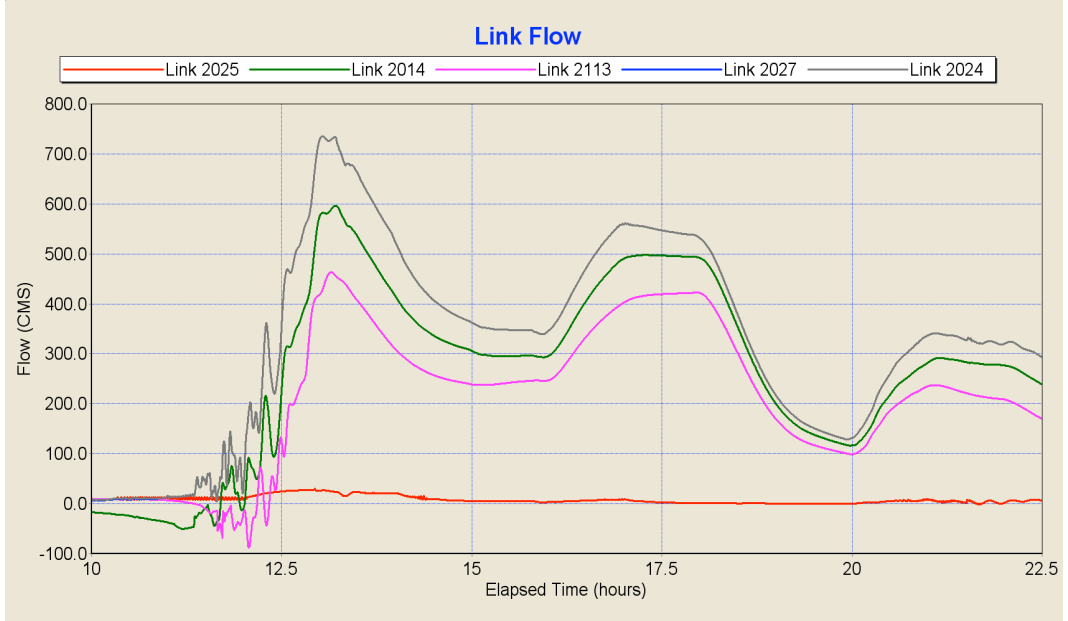

Figure 2.8 Flow discharge traces at the center of various tunnel reaches for the coarse output time (Initial dry bed state, $a=1000$ ).

Figure 2.6 (plot of piezometric depth traces at various conduits) shows several regions of transients. A zoom-in of these regions shows that the peaks and frequency of the pressure oscillations are not well captured. This is because a coarse output time was used for this simulation. For accurately capturing the pressure transients in regions A and B in Figure 2.6, a fine out- 
put time was used for these regions. The simulation results for the piezometric depth for regions A and B in Figure 2.6 (fine output time) are shown in Figures 2.9 and 2.10 , respectively.

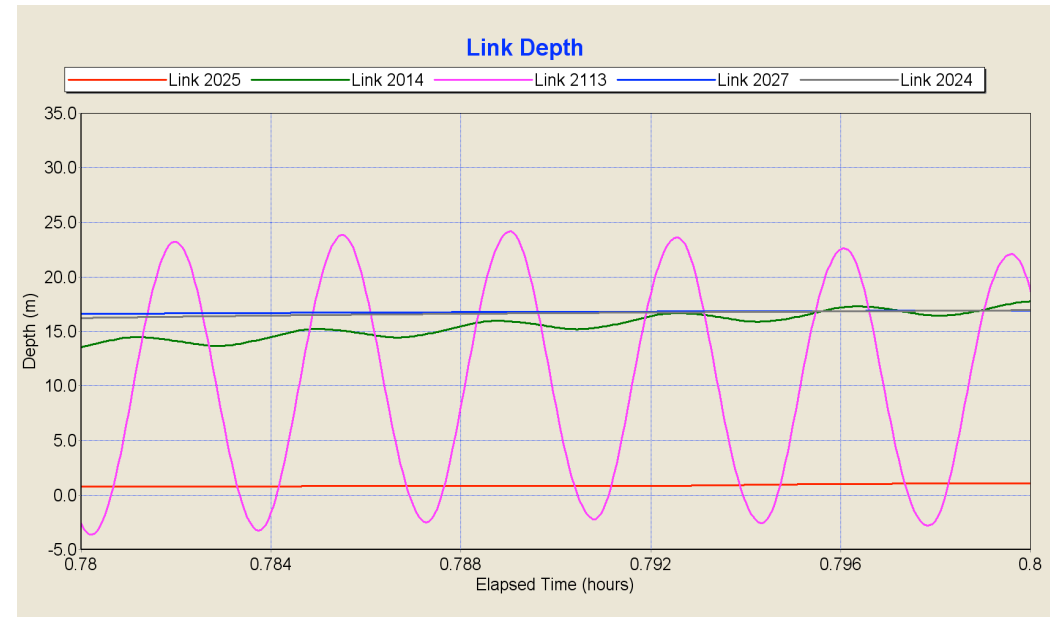

Figure 2.9 Zoom of region A in Figure 2.6 [fine output time].

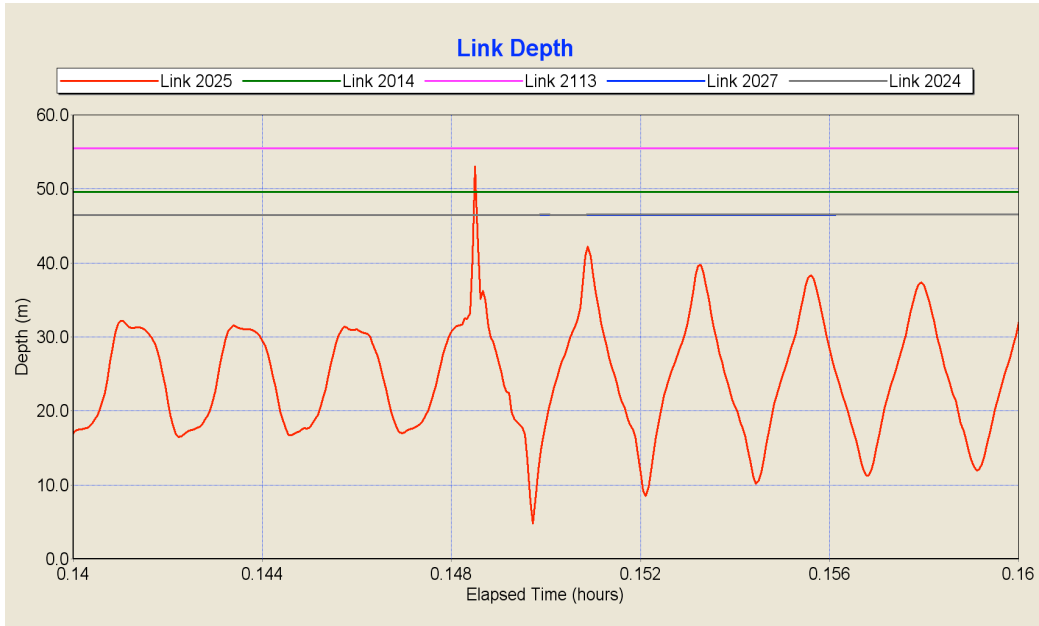

Figure 2.10 Zoom of region B in Figure 2.6 [fine output time].

In the same way as the SWMM model, ITM can generate plots of two variables at any link or node. For instance the plot of depth versus flow dis- 
charge (rating curve) for the link 2027 is shown in Figure 2.11. This figure clearly shows that a rating curve for pressurized flows in transient conditions does not follow the typical rating curve of open channel flows. ITM also generates user-defined tables and a text report that summarizes the results of the simulation. Furthermore, ITM can generate animations for hydraulic grade lines between any two nodes of the system. However, unlike the SWMM model, the plotting resolution of ITM (number of cells per link or reach) is chosen by the user.

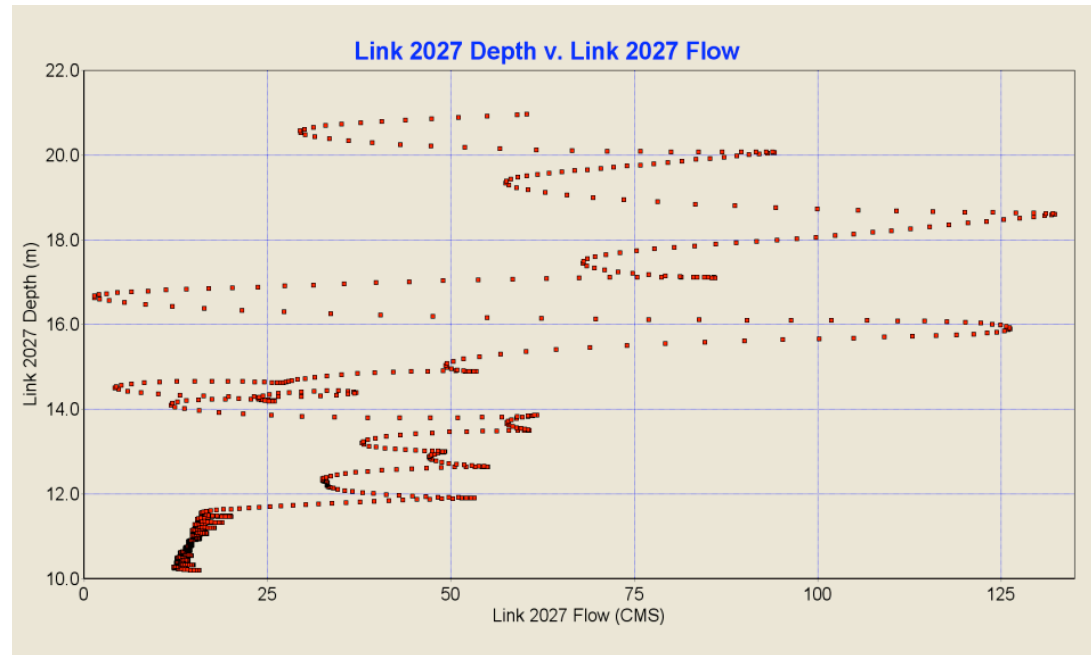

Figure 2.11 Depth versus flow discharge (rating curve) for the link 2027 (mid-way of tunnel) (Initial dry bed state, $a=1000 \mathrm{~m} / \mathrm{s}$ ).

As illustration, Figures 2.12 to 2.15 present hydraulic grade line snapshots between nodes DS15 and the reservoir A at different times. These plots can help to visualize the hydraulic behavior of the system. These plots may also help to identify visually the regions of overflow (when pressure head exceeds terrain level).ITM also generates the text file <name of input file>.INP.debug that is intended for debugging errors. This file is created in the same location of that of the input file. Finally, ITM generates plots for checking conservation of volume in the system. Plots of volume errors in percentage or in cubic meters can be specified. As illustration, Figures 2.16 and 2.17 depict the system volume errors in percentage and in cubic meters, respectively. 
36 Illinois Transient Model: Simulating the Flow Dynamics in CSS Systems

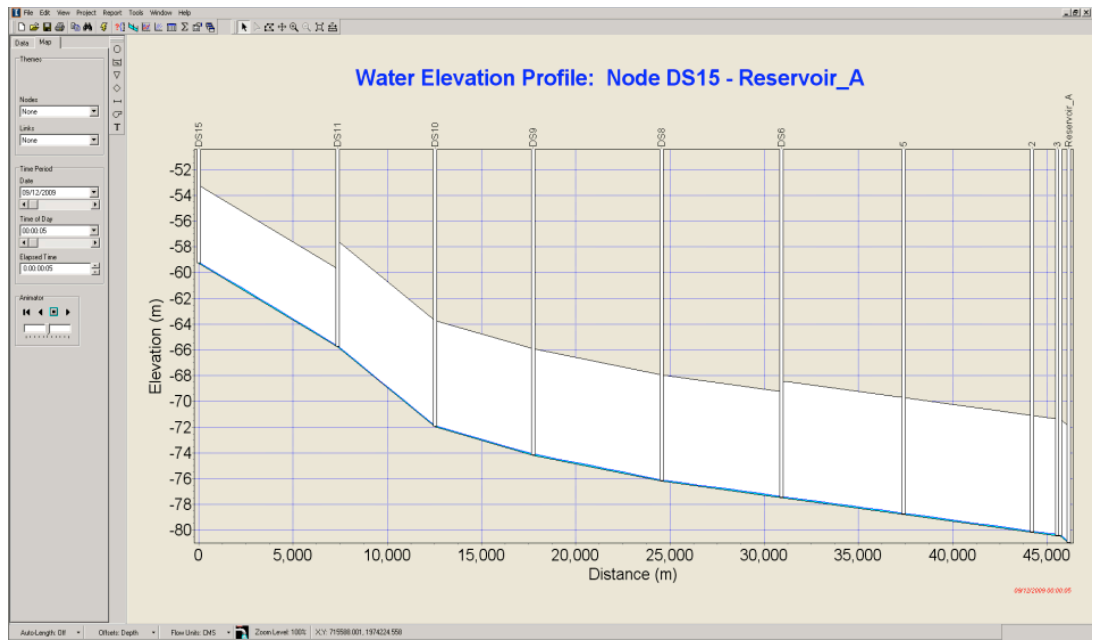

Figure 2.12 Hydraulic grade line snapshot between nodes DS15 and reservoir $A$ after 00:00:05 (Initial dry bed state, $a=1000$ ).

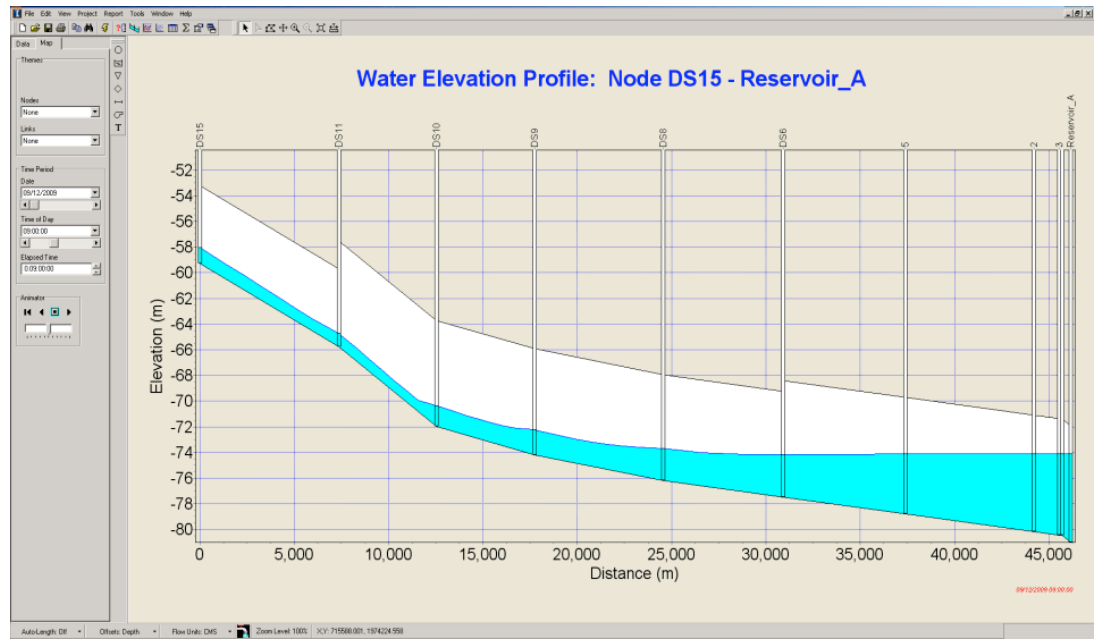

Figure 2.13 Hydraulic grade line snapshot between nodes DS15 and reservoir $A$ after 09:00:00 (Initial dry bed state, $a=1000$ ). 
37 Illinois Transient Model: Simulating the Flow Dynamics in CSS Systems

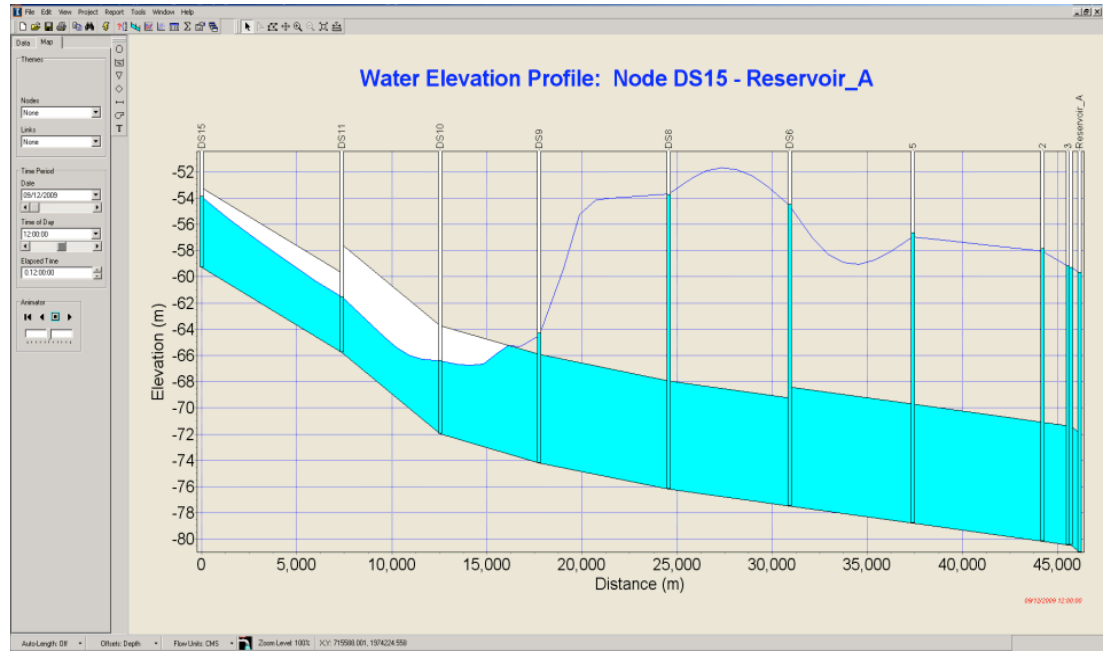

Figure 2.14 Hydraulic grade line snapshot between nodes DS15 and reservoir $A$ after 12:00:00 (Initial dry bed state, $a=1000$ ).

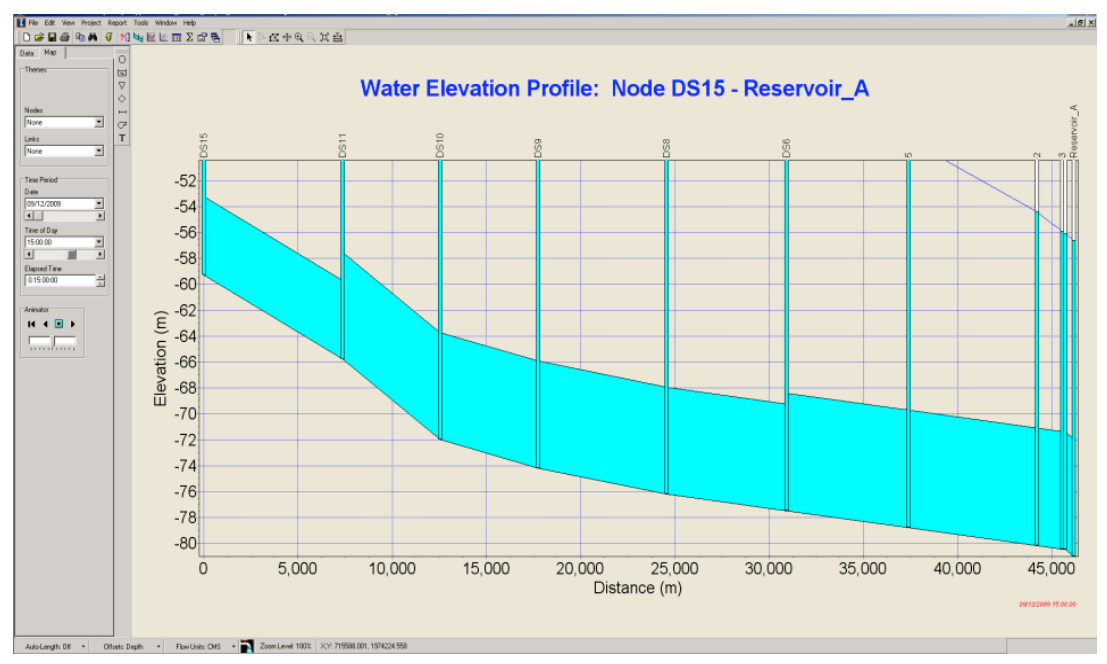

Figure 2.15 Hydraulic grade line snapshot between an upstream node and reservoir A after 15:00:00 (Initial dry bed state, $a=1000$ ). 
38 Illinois Transient Model: Simulating the Flow Dynamics in CSS Systems

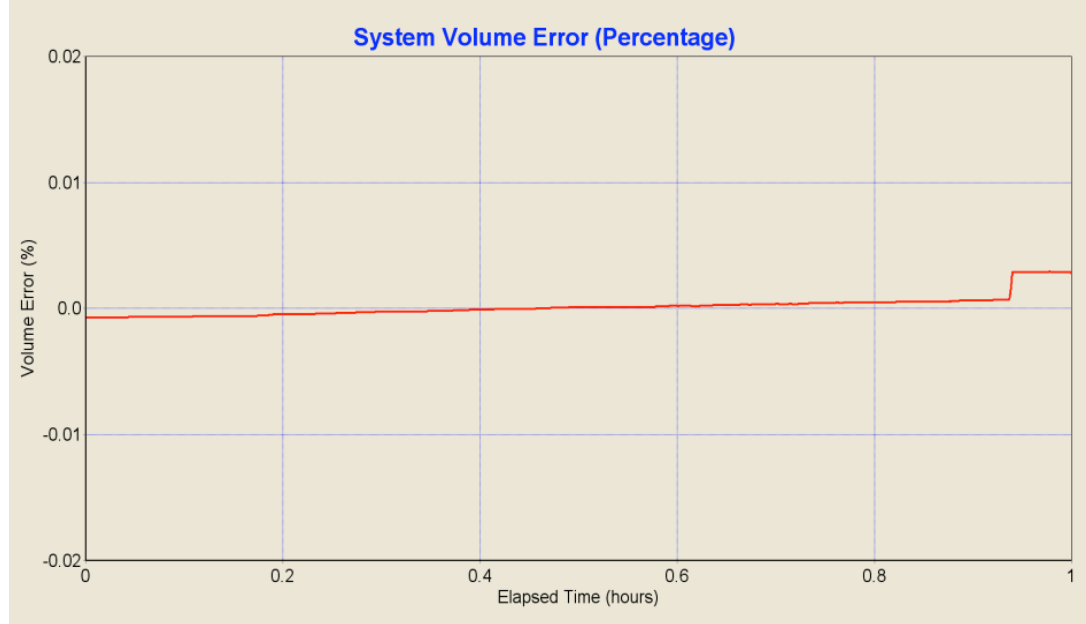

Figure 2.16 System volume error (\%) (fine output time).

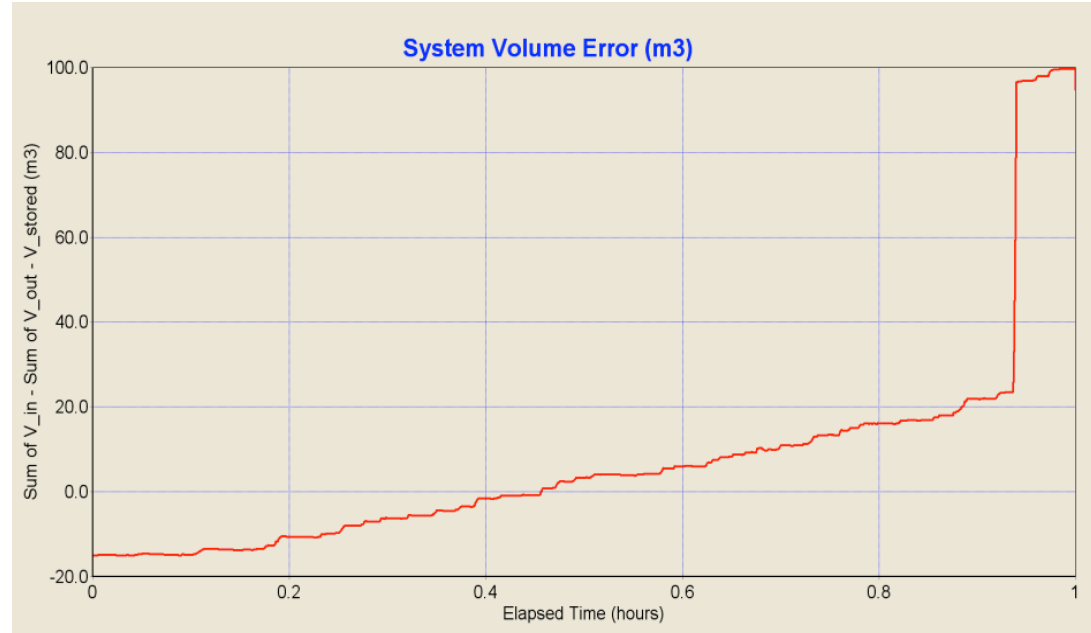

Figure 2.17 System volume error $\left(\mathrm{m}^{3}\right)$ (fine output time).

\subsection{Conclusion}

This chapter presents an overview of the capabilities and features of the recently-developed ITM for simulating the flow dynamics (transient and non-transient conditions) in combined storm sewer systems, ranging from 
dry bed flows, to gravity flows, to partly gravity-partly surcharged flows (mixed flows) to fully pressurized flows (water hammer flows).

\section{Acknowledgments}

The Illinois transient model was originally developed at the University of Illinois at Urbana-Champaign as part of the studies for the tunnel and reservoir plan (TARP) modeling project in Chicago, Illinois. The authors gratefully acknowledge the Metropolitan Water Reclamation District of Greater Chicago for their financial support.

\section{References}

Guinot, V. (2003). Godunov-type schemes. Elsevier, Amsterdam NL.

Leon, A. S. (2006). "Improved modeling of unsteady free Surface, pressurized and mixed flows in storm sewer systems." Ph.D. thesis, Dept. of Civil and Environmental Engng., Univ. of Illinois at Urbana-Champaign, Urbana, IL.

Leon, A. S., Ghidaoui, M. S., Schmidt, A. R., and García, M. H. (2006). "Godunov-type solutions for transient flows in sewers.” J. Hydraul. Eng., 132(8), 800-813.

Leon, A., M.S. Ghidaoui, A.R. Schmidt and M.H. Garcia. 2007. "An Efficient Finite-Volume Scheme for Modeling Water Hammer Flows." Journal of Water Management Modeling R227-21. doi: 10.14796/JWMM.R227-21.

Leon, A.S., Ghidaoui, M.S., Schmidt, A.R., García, M.H. (2008). “An efficient secondorder accurate shock capturing scheme for modeling one and two-phase waterhammer flows.” J. Hydraul. Engng., 134(7), 970-983.

Leon, A.S., Ghidaoui, M.S., Schmidt, A.R., García, M.H. (2009a). “Application of Godunov-type schemes to transient mixed flows." J. Hydraul. Research, 47(2), 147-156.

Leon, A.S., Ghidaoui, M.S., Schmidt, A.R., García, M.H. (2010). "A robust two-equation model for transient mixed flows." J. Hydraul. Research, 48(1), 44-56.

Leon, A.S., Liu X., Ghidaoui, M.S., Schmidt, A.R., García, M.H. (2009b). “A junction and drop-shaft boundary conditions for modeling free surface, pressurized, and mixed free surface-pressurized transient flows." J. Hydraul. Engng., in print.

Leon A. S. and Oberg, N. (2010). "User's manual for Illinois Transient Model-two equation model v. 1.3 (September 2010). A Model for the Analysis of Transient Free surface, Pressurized and Mixed flows in Storm sewer Systems." University of Illinois at Urbana-Champaign.

Song, C.C.S., Cardle, J.A., Leung, K.S. (1983). Transient mixed-flow models for storm sewers. J. Hydraulic Engng. 109(11), 1487-1503.

Toro, E.F. (2001). Shock-capturing methods for free-surface shallow flows. Wiley, Chichester UK.

Wylie, E. B., and Streeter, V. L. (1983). Fluid transients, FEB Press, Ann Arbor, Michigan.

Yuan, M. (1984). Pressurized surges. MSc Thesis. Dept. of Civil and Mineral Engng., Univ. of Minnesota, Twin Cities MN. 
